Musées, Patrimoine et Culture scientifiques et techniques

$144 \mid 2012$

novembre - décembre 2012

\title{
L'apport des sciences participatives à la connaissance de la biodiversité en France
}

Gilles Bœuf, Yves-Marie Allain et Michel Bouvier

\section{(2) OpenEdition \\ Journals}

Édition électronique

URL : http://journals.openedition.org/ocim/1119

DOI : 10.4000/ocim. 1119

ISSN : 2108-646X

Éditeur

OCIM

Édition imprimée

Date de publication : 1 novembre 2012

Pagination : 8-18

ISSN : 0994-1908

Référence électronique

Gilles Bœuf, Yves-Marie Allain et Michel Bouvier, «L'apport des sciences participatives à la

connaissance de la biodiversité en France », La Lettre de l'OCIM [En ligne], 144 | 2012, mis en ligne le 01 novembre 2014, consulté le 01 mai 2019. URL : http://journals.openedition.org/ocim/1119 ; DOI

10.4000/ocim. 1119 


\title{
L'apport des sciences participatives à la connaissance de la biodiversité en France
}

\author{
Gilles Bœuf, Yves-Marie Allain et Michel Bouvier *
}

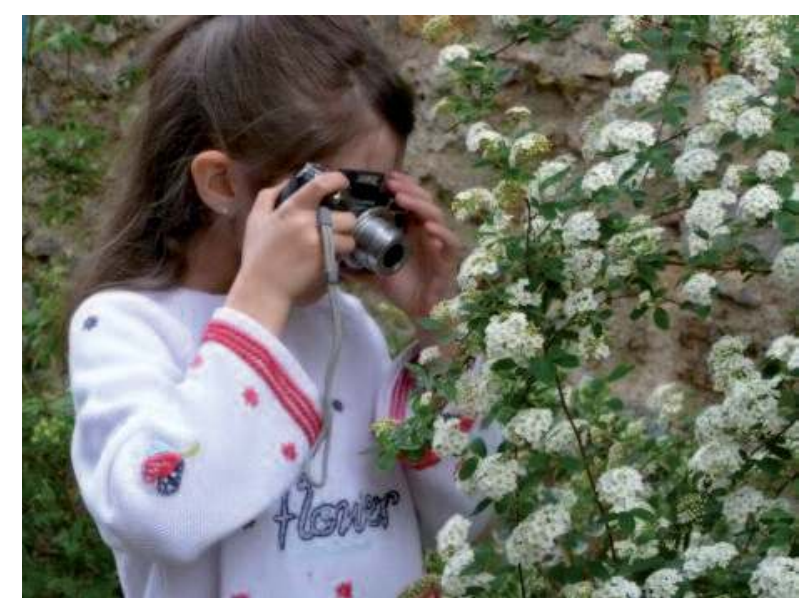

Sophie, observatrice de la biodiversité des jardins (c) Agence Sirius

* Gilles Bœuf est professeur à l'université Pierre et Marie Curie, président du Muséum national d'Histoire naturelle et membre du Conseil scientifique du Patrimoine naturel et de la Biodiversité auprès du ministère de l'Écologie, du Développement durable, des Transports et du Logement bœuf@mnhn.fr

Yves-Marie Allain et Michel Bouvier sont membres du Conseil général de l'Environnement et du Développement durable/commission permanente des ressources naturelles au ministère de l'Écologie, du Développement durable, des Transports et du Logement
Issues d'un rapport remis au ministère de I'Écologie, au début de l'année $2012^{\text {(1), }}$ ces réflexions rassemblent une série $d^{\prime}$ interrogations fondamentales et récurrentes sur le fond et sur les aspects méthodologiques des sciences participatives : elles insistent sur l'indispensable caractère scientifique de l'objectif, la nécessaire compétence scientifique des participants et suggèrent une coordination générale et centralisée laissant une place à l'aspect spontané $d^{\prime}$ un certain nombre d'initiatives.

\section{Introduction}

Les Nations Unies ont déclaré l'année 2010 «Année internationale de la biodiversité» : pourquoi ? En 2002, lors de la Conférence internationale de Johannesburg, il avait été décidé de freiner l'érosion de la biodiversité pour 2010 et l'Europe avait surenchéri : «stopper cette érosion », nous en sommes encore loin en 2012. Au contraire, la situation n'a jamais été si préoccupante (MEA, 2005, Barbault, 2006, Butchart et al., 2010, Barnosky et al., 2011, He et Hubbell, 2011).

Les impacts de l'humanité sur son environnement au cours de l'histoire récente (50 000 dernières années) sont plus ou moins bien documentés dans la littérature scientifique, mais quelques solides données existent. Bien entendu, on pourra toujours dire, et pertinemment, que les variations climatiques fréquentes au cours des derniers millénaires ont fortement contribué aux mouvements de faunes et flores et à leur 
extinction totale ou partielle (Bœuf, 2008 ; Blondel et al., 2010). Il n'en demeure pas moins réel que l'humain porte une forte responsabilité dans nombre de situations (CSPNB, 2007, 2008, 2012). S'il a souvent apporté les conditions de la "goutte d'eau qui fait déborder le vase », il est aussi souvent seul en cause, en particulier dans tous les écosystèmes insulaires et même sur le grand continent australien, qui n'a pas vécu de bouleversement climatique depuis longtemps. Durant des centaines de milliers d'années, les Homo n'ont pas plus impacté leur environnement qu'un mammifère omnivore de taille comparable (Bœuf, 2010), un sanglier par exemple. Mais en fait, dès la domestication du feu par H. erectus vers 800000 ans av. J.C., l'influence a été de plus en plus marquée. Rapidement (et nous suivons bien volontiers Lévêque et Mounolou en 2001, quand ils précisent que le « mythe $d u$ bon sawvage » en prend un coup !), l'espèce " sapiens » de plus en plus technique, ingénieuse, intelligente, inventive et agressive commence à avoir un impact inquiétant sur les milieux et à dégrader son environnement, souvent de manière irréversible pour la biodiversité. Ceci s'accélère considérablement lors de la « révolution néolithique » vers 14000 ans av. J.C. avec le premier «baby boom » lié à l'arrêt de l'exclusif nomadisme et surtout des premières réussites de l'élevage et de l'agriculture. Les humains s'organisent en villages puis villes, vers le cinquième millénaire, et se protègent mieux collectivement. La troisième "révolution » est industrielle, avec des besoins de plus en plus insensés en matières premières et combustibles fossiles, besoins qui s'accélèrent avec une population humaine qui a doublé sur 50 ans. Cette époque a été proposée comme l'« anthropocène » par Crutzen et Stoermer (2000).

Il est bien clair que la biodiversité ne saurait être représentée dans sa totalité par le seul inventaire et la description des espèces vivantes peuplant un écosystème particulier. Ceci est la diversité spécifique. La biodiversité est beaucoup plus que cela, c'est tout l'ensemble des relations établies entre les divers êtres vivants, entre eux et avec leur environnement (Bœuf, 2008). Elle a aussi été définie comme étant toute linformation génétique contenue dans chaque unité élémentaire de diversité (Blondel, 2005), un individu, une espèce, une population, un écosystème. En étendant le sujet scientifiquement, nous mettons globalement quatre aspects dans le terme «biodiversité » aujourd'hui (Lévêque et Mounolou, 2001 ; Bœuf, 2008) :

- l'étude des mécanismes biologiques fondamentaux permettant d'expliquer la diversité des espèces et leurs spécificités et nous obligeant à davantage « décortiquer » les mécanismes de la spéciation et de l'évolution;

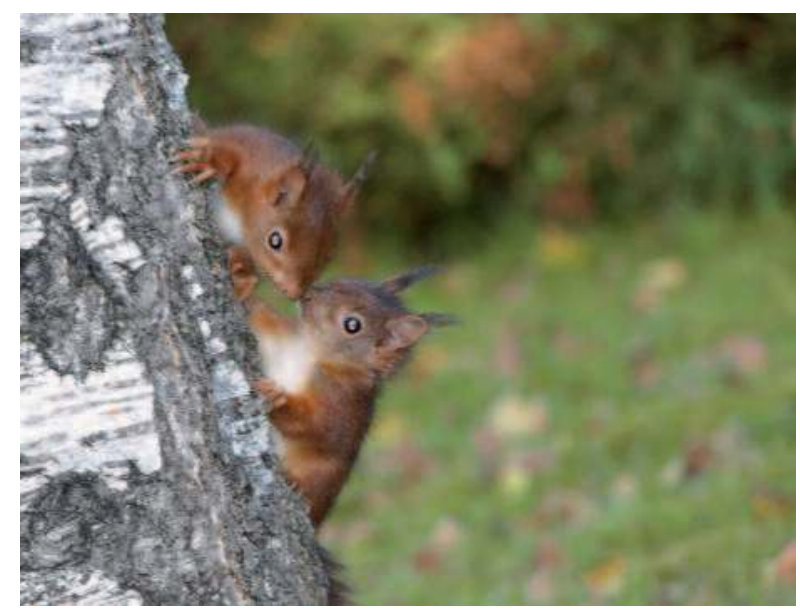

« 50000 observations pour la forêt » : les Écureuils roux () Family Gouhenant

- les approches plus récentes et prometteuses en matière d'écologie fonctionnelle et de bio-complexité, incluant l'étude des flux de matière et d'énergie et les grands cycles biogéochimiques ;

- les travaux sur la nature « utile » pour l'humanité dans ses capacités à fournir des aliments, des substances à haute valeur ajoutée pour des médicaments, produits cosmétiques, des sondes moléculaires ou encore à offrir des modèles originaux et pertinents pour la recherche fondamentale et finalisée, afin de résoudre des questions agronomiques ou biomédicales (Bœuf, 2008) ;

- la mise en place de stratégies de conservation pour préserver et maintenir un patrimoine naturel constituant un héritage naturellement attendu pour/par les générations futures. La biodiversité en fait, c'est le vivant dans toute sa complexité, c'est la fraction vivante de la Nature. En estimant les vitesses d'évolution, en tentant de prédire les trajectoires possibles et en planifiant les mécanismes à l'avance, nous pourrions sans doute fortement réduire l'impact de l'humain sur les espèces et les écosystèmes et sérieusement améliorer les coûts économiques et sociaux de nos activités sur la nature. En 2001, Palumbi concluait «... l'humain est devenu la plus puissante force évolutive de la $\mathrm{Na}$ ture... ». Le climat change et la biodiversité doit s'adapter, tout est une question de capacité et de vitesse de réaction (Thomas et al., 2004, Barriopedro et al., 2011).

\section{Les sciences participatives}

Pour suivre cette évolution, la collecte des informations nécessaires à ces observations implique de mobiliser un très grand nombre de « petites mains " pour obtenir des résultats significatifs (Mathieu, 2011). Ces 
actions citoyennes permettent de mieux connaître la répartition et l'évolution des populations des espèces vivantes, de mieux comprendre les raisons de leur déclin ou de leur expansion, de mieux définir les indicateurs de mise en évidence de ces phénomènes et enfin de mieux sensibiliser nos citoyens aux questions environnementales (Happe, 2010). Elles s'adressent à tous, du naturaliste le plus confirmé au simple amoureux de la Nature. Les seules institutions scientifiques et les services de l'État dédiés à la gestion de l'environnement ne suffisent pas et les moyens sont limités. De nombreuses sciences naturalistes ne peuvent se passer d'un grand volant d'amateurs, répartis et disséminés sur le territoire, pour atteindre une masse critique que les sciences physiques par exemple compensent par l'utilisation de grands équipements dans un espace confiné (Charvolin, 2009). De plus la participation citoyenne est vitale pour maintenir une pression constante sur l'urgence et la nécessité impérative de réagir et pour un établissement en commun de l'état de la situation ainsi qu'une responsabilisation collective.

Dans cet objectif de connaissance de la biodiversité et de son évolution, quel pourrait ou devrait être l'apport des sciences participatives ? Quelques ouvrages avaient déjà été publiés en France sur le sujet, le rapport de M. Legrand en 2010, un livret de Tela Botanica en 2009, un autre de l'IFRÉE en 2011, avec des listes d'associations.

Les expressions de sciences participatives, sciences citoyennes, sciences collaboratives... sont récentes, la participation du public ou du moins de certains publics à la prospection, à la récolte de données, d'échantillons, à la connaissance... n'est pas d'hier et remonte en Europe, au moins pour la botanique, au XVIe siècle. Dès la prise de possession d'un territoire outre-mer par l'une des nations européennes, très vite vont se trouver mis à contribution les gouverneurs, le personnel consulaire, les ecclésiastiques, missionnaires, militaires, médecins, chirurgiens, aventuriers divers et variés... Certains seront des naturalistes de qualité, explorant des zones reculées, peu ou encore jamais prospectées. Bien que n'appartenant pas à une institution scientifique « officielle », certains possèdent très souvent une solide culture scientifique à caractère assez universel, qui leur permet de développer un fort sens critique, basé sur d'excellentes qualités d'observation et une passion forte.

Au XVIIIe siècle, une Ordonnance du 9 septembre 1726 de Louis XV enjoignait les capitaines de navire d'«apporter des graines et plantes des colonies, des pays étrangers... ». Pour obtenir des plantes, Duhamel du Monceau fait appel à de très nombreux correspondants

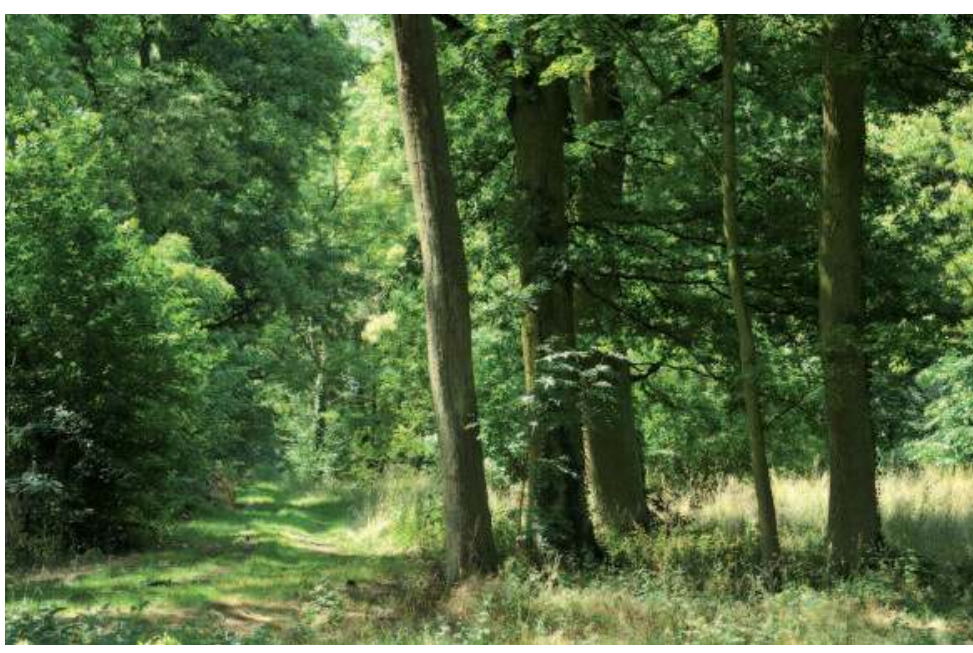

L'Arboretum de Chèvreloup (c) François Grandin/MNHN

que sont les médecins du roi, les gouverneurs en place dans les possessions françaises d'Afrique et d'Amérique ou les consuls installés au Moyen-Orient. Au XIX ${ }^{\mathrm{e}}$ siècle et au début du XX $\mathrm{X}^{\mathrm{e}}$ siècle, plusieurs opuscules sont édités par le Muséum national d'Histoire naturelle, dont l'un au titre explicite : Instruction pour les voyageurs naturalistes et pour les employés dans les colonies sur la manière de recueillir, de conserver et d'envoyer les objets d'histoire naturelle. Des listes de plantes et autres objets à rapporter sont proposées par les professeurs du Muséum.

À compter de la seconde moitié du XIXe siècle, en France, les sociétés savantes se développent et face à la diversité des mondes naturels, vivant et inerte, les niveaux des connaissances progressent à une vitesse exponentielle, et rapidement elles se spécialisent dans un domaine naturaliste plus ou moins spécifique : botanique, entomologie, ornithologie, géologie, minéralogie, paléontologie... Durant cette même période, dans les grandes villes françaises sont créés des muséums régionaux d'Histoire naturelle, et se développent des sorties naturalistes dans toutes les provinces. Les publications locales ou régionales - botanique, entomologie, géologie... - sont révélatrices du travail d'inventaire effectué par ces réseaux, y compris sur des groupes assez délaissés actuellement comme les lichens, les mousses (bryophytes), les fougères (ptéridophytes) ou encore les « algues » marines... Les réseaux constitués sont bien identifiés avec des réunions à caractère scientifique assez fréquentes, hebdomadaires ou mensuelles. Ils font avancer la connaissance dans de très nombreuses régions qui ne possèdent pas encore d'université. Sur le reste du 
territoire, le réseau, dense, des instituteurs, des ecclésiastiques, des médecins ruraux, des vétérinaires de campagne... va être également l'un des facteurs de diffusion des sciences, de la formation à l'observation naturaliste, de l'encadrement des collecteurs bénévoles, de la collecte de ces informations, des vérifications du bien fondé des inventaires et de leur publication. Malgré cette abondance de prospecteurs, une très grande partie des observations et données récoltées ont disparu. Souvent inscrits sur des carnets plus ou moins lisibles pour une tierce personne, ces relevés plus ou moins complets, avec un cadre méthodologique souvent personnel, non reproductible, pas toujours fiable, sont bien souvent très partiels et difficilement exploitables. Dans les dernières décennies du $\mathrm{XX}^{\mathrm{e}}$ siècle, pour des raisons diverses, les associations naturalistes " académiques » ont perdu de leur attrait, les sciences de l'observation ont quelque peu périclité, étant considérées comme peu novatrices, trop descriptives, voire sans intérêt par les commissions d'évaluation des «sciences de la vie» et par certains comités éditoriaux de grandes revues scientifiques.

À partir des années 1960, le travail de fond effectué par de nombreuses associations de défense de la nature a permis d'enrayer ce déclin, de renverser la tendance et de recréer des réseaux de naturalistes actifs, effectuant comptages, recensements, observations surtout chez les botanistes, ornithologues et entomologistes. Si les techniques de relevés des observations n'ont guère évolué sur le terrain, la banalisation des outils modernes de communication dont Internet, va profondément modifier les possibilités de diffusion des données et leur mise à disposition pour tous. Devant cette facilité technique, avec des outils informatiques plus conviviaux, une rigueur méthodologique certaine va s'avérer nécessaire pour tous les

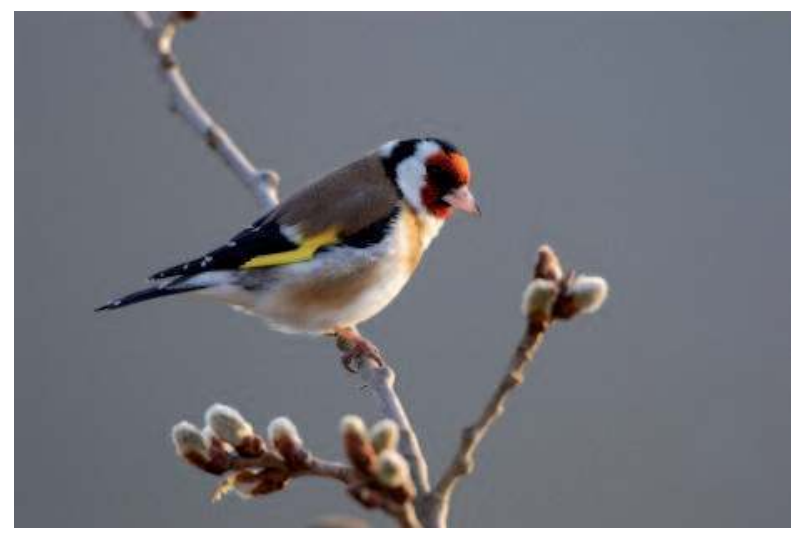

L'observatoire des oiseaux des jardins : le Chardonneret... (c) F. Cahez/LPO acteurs, dont les « amateurs » (souvent « très éclairés»), avec le respect des protocoles d'observation et de récolte des données tant dans les objets observés que dans la manière de les traiter afin de pouvoir les exploiter et en tirer des conclusions les plus pertinentes et étayées. Pour illustrer le lien entre l'histoire des simples inventaires et une reprise actuelle, autorisant à proposer des hypothèses sur la dynamique des populations, rappelons l'exemple de la traversée des Pyrénées (sur mandat napoléonien) et l'herbier d'A. de Candolle, qui ont donné lieu à une répétition comparative 200 ans plus tard, à l'initiative d'une association (Terranoos) et à la parution d'un bel ouvrage.

Des articles récents $(2010,2011)$ dans des revues de vulgarisation comme Terre sawvage (France) ou la Revue durable (Suisse) communiquent sur les sciences participatives, notamment sur les principales actions pilotées par le Muséum national d'Histoire naturelle. Par ses circulaires, le ministère en charge de l'Écologie, incite également les services déconcentrés à s'appuyer sur des démarches participatives pour la mise en œuvre de certaines politiques dont celle de la stratégie nationale de création d'espaces protégés, voire de la stratégie nationale pour la biodiversité. En outre, deux colloques sur les réseaux collaboratifs et les sciences citoyennes ont été organisés à Montpellier en 2009, le premier « Réseaux collaboratifs et sciences citoyennes » sous l'égide de l'association Tela Botanica, le second "Le réveil du dodo » lors des Journées francophones de la biologie de la conservation.

Ce phénomène de participation du public n'est pas propre à la France. À l'étranger, des expériences, dont celles de la Grande-Bretagne, ou celles des «Citizen Sciences » nord-américaines créées vers 1970, montrent la très forte implication du public, avec des taux de participation qui peuvent laisser pantois par rapport aux dispositifs français. Aux États-Unis, le

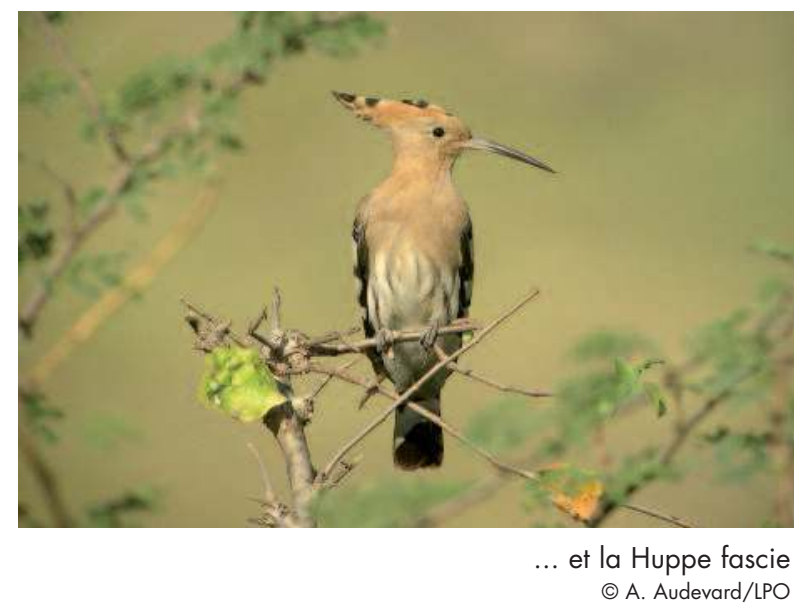


Christmas Bird Count, créé en 1900 et piloté par la Société Audubon, est régulièrement reconduit depuis 1900 (Mathieu, 2011) : 10000 «observateurs participatifs » ont comptabilisé 63 millions d'oiseaux dans le cadre d'un programme qui a souligné, sur une quarantaine d'années, une régression de $68 \%$ de 20 espèces communes. Le programme français du suivi temporel des oiseaux communs (STOC) en est l'équivalent depuis 1989.

Dans un monde sacrifiant beaucoup à la Science, comment rendre justice à des approches cognitives liées à une approche en "plein air », adopter une posture qui jette les bases d'une coexistence entre « amateurisme » et «professionnalisme »? Du fait qu'il n'est ni totalement ignorant (la plupart du temps, bien loin de là), ni totalement professionnel, l'« amateur » trouble l'image de la science « normale » confinée et reproductible (Charvolin, 2009). Il est clair aujourd'hui que nous manquons de travaux en sociologie sur une réflexion sur ces aspects.

En fait, la question essentielle aujourd'hui est un encouragement clair et non équivoque sur la sensibilisation d'un public plus large à la thématique de la biodiversité et de la connaissance de la nature en créant un appui au développement d'un tel mouvement comme moyen complémentaire de la politique de recherche et de gestion dans ces domaines (Couvet et al., 2008). Une certaine « labellisation », éventuellement par voie réglementaire, doit en garantir l'intérêt tant au plan scientifique que pour les applications que l'on peut en tirer jusqu'à des propositions de modes de gestion de notre environnement plus pertinentes et durables. Nous nous devons cependant d'insister sur quelques aspects.

\section{Le caractère scientifique de l'objectif}

Divers programmes peuvent avoir pour objet un simple inventaire d'espèces. L'inventaire implique une connaissance scientifique de base pour sécuriser l'identification. Toutefois le résultat reste limité en termes de recherche. Il peut viser la présence ou l'absence d'une espèce, ou la quantifier (modèles d'abondance dans le temps). Il peut cadrer un domaine géographique large (commune par exemple, dans l'établissement des atlas communaux lancés en 2010 par le ministère en charge de l'Écologie) ou exiger une géo-localisation plus précise, en relation potentielle avec des caractères de milieu. On a là une progression de la démarche éventuellement de hors-champ à science citoyenne, puis participative. Un inventaire renouvelé, ou un observatoire permanent ont déjà plus de consistance en fournissant une donnée d'évolution. On ne peut donc les exclure du sujet.

\section{La compétence scientifique des participants}

La validation scientifique des données recueillies est nécessaire. Au départ, les mobilisations bénévoles impliquent souvent des « amateurs éclairés » (ce sont aussi souvent d'anciens professeurs ou chercheurs, partis à la retraite), et souvent connus des chercheurs en activité, ce qui amène la confiance a priori. Dans divers programmes, une formation peut être apportée. SPIPOLL (suivi photographique des insectes pollinisateurs) fournit par exemple en ligne un outil de détermination, même si la qualité première recherchée est celle de la photographie. L'utilisation des données est aussi raisonnée par des tests de vraisemblance ou de cohérence qui peuvent amener à une sélection des participants, même si elle est «sociologiquement délicate ». On peut évoquer certains outils permettant une collecte plus normalisée de données, par exemple les bat-boxes (enregistrant les ultrasons caractéristiques des diverses espèces de chiroptères) voire des reconnaissances automatisées d'oiseaux sur enregistrement. Ces outils ont leur limite mais les marges de progrès sont possibles. Une polémique assez violente avait été déclenchée après le lancement de SPIPOLL en mai 2010 avec un groupe de chercheurs belges spécialisés dans les insectes pollinisateurs. Ces derniers ont même accusé le Muséum de faire de la "pseudoscience »! Tout ceci a atteint un point d'orgue dans un numéro de la revue La Recherche au printemps 2011. En fait, il est clair que le millier d'insectes pollinisateurs en France (sur les 2 millions d'espèces connues, 250000 sont des

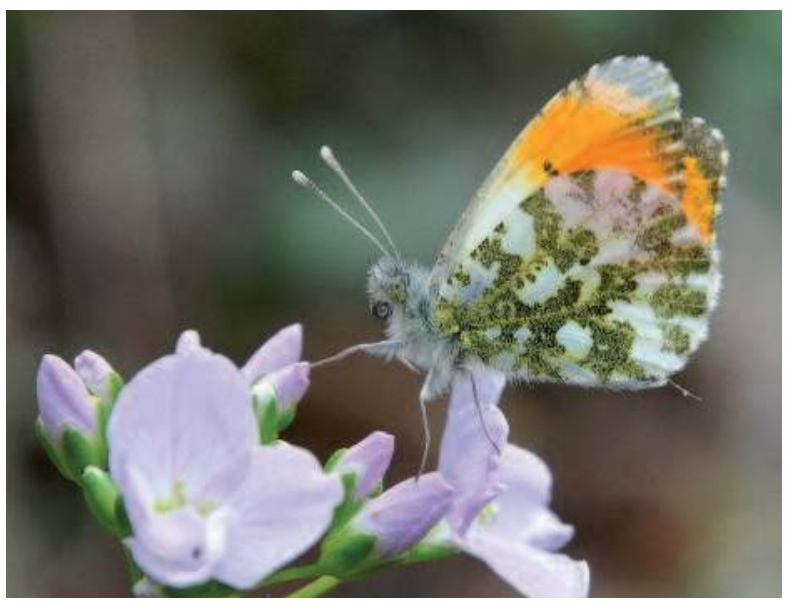

Le suivi des insectes pollinisateurs : le Papillon Aurore (c) O. Dulmet, observateur SPIPOLL 
pollinisateurs) représente beaucoup plus de difficultés d'identification que les quelques 100 «oiseaux communs ", 30 chauve-souris ou la vingtaine de "papillons de jardin », recensés dans les études précédentes. Mais par ailleurs, donnons-nous quelque temps pour faire une analyse impartiale du « retour » de SPIPOLL après deux années d'existence. STOC avait aussi été très critiquée lors de son lancement et nul n'envisage aujourd'hui de remettre en cause cette action. Un article vient juste d'être accepté dans la revue PLoS ONE grâce aux efforts de milliers de « participatifs » à SPIPOLL (Deguines et al., 2012).

\section{Une coordination générale et centralisée ?}

Il serait souhaitable que la France puisse tendre vers une vision globale des diverses sollicitations du public pour améliorer les connaissances. Nous avons estimé le nombre actuel d'associations pouvant prétendre en France à la dénomination de «sciences participatives » à environ 200. Mais, dans le même temps, il ne faut surtout pas étouffer la spontanéité de certaines initiatives, ni proposer d'homogénéiser les techniques à mettre en œuvre. Mais une meilleure connaissance des initiatives et un partage des données pourraient être envisagés afin d'éviter les programmes redondants ou les groupes taxonomiques « orphelins ». Dans ce cas une grille d'évaluation du caractère de sciences participatives serait utile. L'idée de la création d'une structure inspirée du Citizen Science Alliance pourrait permettre de jouer ce rôle de partenaire fédératif national pour toutes les initiatives françaises. Afin de ne pas créer un nouvel instrument, un tel rôle ne pourrait-il pas être tenu par le Muséum national d'Histoire naturelle ?

\section{Conclusions}

Dans une interview donnée à deux collègues (Gosselin et al., 2010) par Romain Julliard (Julliard, 2010), l'un des animateurs essentiels des sciences participatives au Muséum, les questions majeures posées étaient :

- Pourquoi faire appel au grand public pour suivre l'évolution de la nature (biodiversité, mais aussi phénomènes physiologiques, comme dans l'observatoire des saisons)?

- Quel est l'historique des suivis participatifs ? Dans quel pays est-ce le plus développé ?

- Comment se fait le choix des taxons à suivre ?

- Ces suivis suscitent-ils un engouement réel et durable de la part des scientifiques et de la part du public?

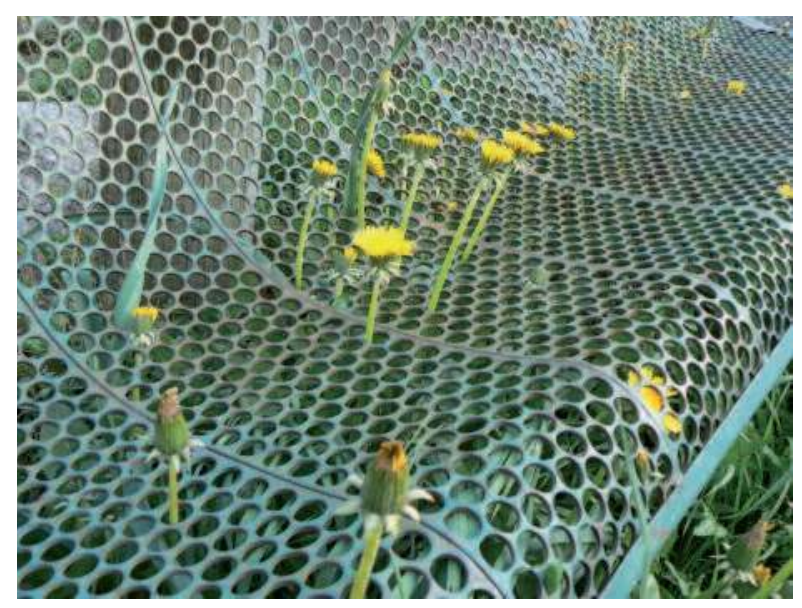

La biodiversité en ville, "Sauvages de ma rue »:

le Pissenlit urbain

๑) J. Neuschwander/CC-by-SA

- N'y a-t-il pas de forts «effets observateurs » et des risques d'erreurs si les observateurs sont novices? Si oui, comment les corrige-t-on et comment analyser les données?

- Comment maîtriser la représentativité de l'échantillonnage sur un territoire donné, lorsque les points d'observation sont contraints par le lieu d'habitation des observateurs?

- Peut-on espérer des réseaux mixtes professionnels/ amateurs, les professionnels «bouchant les trous » du plan d'échantillonnage?

- Peut-on envisager un réseau « professionnel » pour évaluer l'efficacité des politiques publiques de protection de la biodiversité ?

- Les suivis sont-ils considérés comme un enjeu fort par les décideurs et plus généralement par la société ? - Les protocoles sont-ils cohérents entre pays ?

- Quel lien et quelles différences entre les suivis participatifs et le SINP (Système d'informations sur la nature et les paysages) ?

- Le ministère en charge de la Recherche ou le CNRS réfléchissent pour leur part au montage d'observatoires de recherche en environnement. Ces observatoires permettraient-ils d'évaluer les politiques publiques de conservation de la biodiversité ?

De cet ensemble de questionnements, logiques et techniques, il ressort des interrogations fondamentales et récurrentes «sur le fond » des sciences participatives, et sur des aspects méthodologiques.

En Hollande comme en Angleterre, dans les pays de culture anglo-saxonne en général, les gens sont très impliqués dans la vie collective, plus que dans les pays de culture latine. Beaucoup d'associations remplissent des fonctions de services publics, et ce, dans tous les domaines. Dans le domaine naturaliste, les 
observateurs sont donc beaucoup plus faciles à mobiliser qu'en France, quels que soient les taxons. Le mouvement associatif est plus fort dans ces pays (par exemple la Royal Society for the Protection of Birds (RSPB) compte plus d'un million de membres, là où la LPO en France en compte un peu plus de 45 000. Bien que toute généralisation soit toujours sujette à caution, la différence de culture se traduit aussi dans la grande confiance que les observateurs anglo-saxons font dans les protocoles et le projet collectif de suivi. Dans les pays de culture latine en revanche, l'observateur est plus méfiant (Julliard, 2010) et se pose trois questions avant de s'engager :

- Est-ce que je ne risque pas d'être « instrumentalisé » ?

- Ce projet de suivi est-il utile ?

- Est-ce que je suis utile pour ce suivi ?

Dans une publication récente, Couvet et ses collaborateurs (2011) résument la situation : «Afin de développer un système complet d'observation de la biodiversité, les forces et les limites des différentes méthodes d'observation doivent être comparées. Nous examinons la pertinence des suivis documentant régulièrement, sur un grand nombre de sites, sur un large territoire, les caractéristiques de nombreuses espèces d'une communauté (abondance des espèces, phénologies...), caractérisant la variation spatiale fine de la biodiversité. De tels suivis doivent s'appuyer sur les sciences participatives, apportant d'autres avantages. La mise en relation des variations observées avec les facteurs de changements globaux apporte des connaissances en macro-écologie et en écologie des communautés. Ces suivis extensifs peuvent estimer des effets diffus, facilitant une utilisation raisonnée du principe de précaution. En complément des études approfondies menées sur quelques sites, ces suivis sont la base nécessaire à la construction de scénarios de biodiversité ».

Nous connaissons, aujourd'hui déposées et archivées dans les musées d'Histoire naturelle, plus de 2 millions d'espèces et nous continuons à en décrire entre 16 et 18000 par an, tout en sachant quilil nous en reste de 10 à 30 fois plus à découvrir (Mora et al., 2011 ; Bœuf, 2011). Nous n'en n'avons plus le temps, nous en aurons perdu la moitié à la fin de ce siècle. Il n'est pas exceptionnel de voir une espèce disparaître entre sa première collecte et sa description. Et nous avons vu que la biodiversité est bien autre chose que le seul archivage d'espèces ! La question de la restauration (quand elle est possible) des habitats est essentielle (Ray-Benayas et al., 2009 ; Vila et al., 2011). Dans leur rapport récent pour le ministère en charge de l'Environnement, Le Maho et Boucher

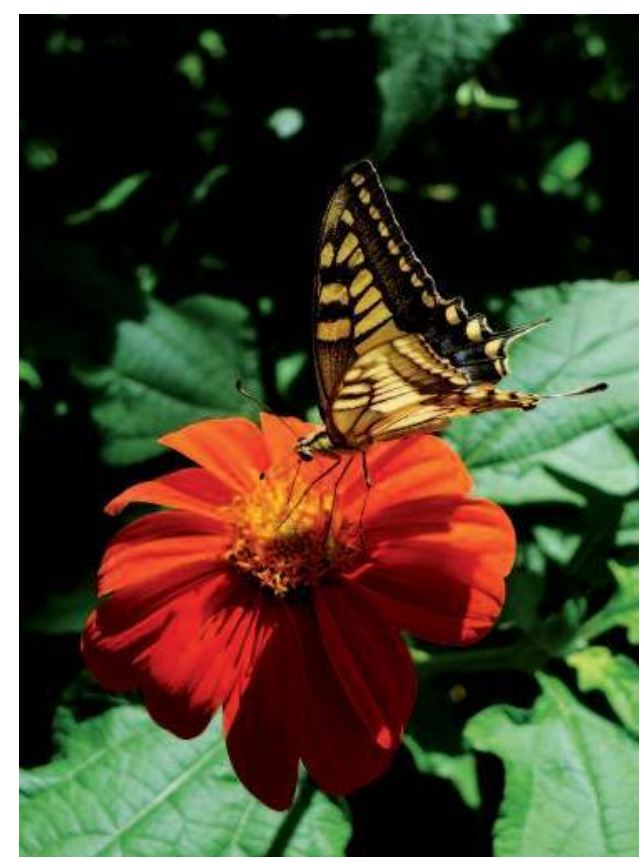

L'observatoire de la biodiversité des jardins : le Papillon Machaon ○) Magali Evanno

(2011) reviennent sur la faiblesse des travaux en France concernant le suivi à long terme de l'évolution de la biodiversité. Suite à une proposition du ministère, ils analysent l'intérêt de la création d'une "Agence de l'expertise » de la biodiversité. Aujourd'hui le développement des sciences participatives est un fait de société et le combat permanent pour sauver la biodiversité en est imprégné. Au niveau des collectivités, des organisations institutionnelles diverses, des services du ministère en charge de la protection de la nature, ces sciences participatives sont présentes et doivent très sérieusement être prises en considération. Lors du premier Congrès commun des Réserves naturelles de France et des Conservatoires d'espaces naturels, à Aix-les-Bains en avril 2011, leur importance a encore été soulignée.

Les données collectées se révèlent de plus en plus pertinentes et utiles et amènent aujourd'hui à la publication d'articles fondateurs, dans de très grandes revues, déterminants pour une meilleure prise en compte des grands changements présents. À ce sujet ( 1,5 millions d'heures d'observations!), le papier de janvier 2012 dans Nature Climate Change, sur les migrations liées à la «traque climatique » des oiseaux et des papillons (Devictor et al., 2012) en Europe de l'Ouest, est emblématique. Lors de la mise en place de la Convention sur la diversité biologique (CBD), le besoin d'indicateurs « mesureurs » de l'évolution de la biodiversité s'était rapidement fait 
sentir et nous bénéficions aujourd'hui d'une vingtaine d'indicateurs pour évaluer les cibles fixées. Mais seul l'un d'entre eux « Tendances de l'abondance et de la distribution des espèces » n'est pas directement relié à une exploitation économique. En France, les données dans ce domaine sont totalement liées aux «participatifs » et leur rôle est fondamental. Une étude récente (Levrel et al., 2010) a même démontré que leur apport pouvait se chiffrer (temps passé, qualité du travail fourni, constance...) entre 0,7 et 4,4 millions d'euros par an pour l'administration et la recherche. En fait, ces participations à la fourniture de données relatives à la biodiversité sont essentielles. Daniel Mathieu (2010) propose même de passer du «scientifique citoyen » au " citoyen scientifique»! L' " amateur » impliqué est un "citoyen ordinaire», doté de compétences particulières, qu'il va mettre au service d'une cause publique, hors cadre professionnel. Les documents issus de ces partenariats désirés peuvent être très performants, voir par exemple les cartes de répartition de plantes en France établies grâce au réseau de Tela Botanica (14 000 participants), les suivis sur les oiseaux de la Ligue de Protection des Oiseaux ou encore de l'Observatoire des saisons. Certains scientifiques ont encore des réticences (voir « l'affaire SPIPOLL » évoquée précédemment) à utiliser des données non issues de laboratoires reconnus mais cela change en matière de diversité biologique et de travaux fréquents et nombreux sur le terrain. Il persiste la question de la validation des données et parfois de méthodologies expérimentales délicates à appliquer sur le terrain. C'est ici qu'intervient la question dominante de la formation des collecteurs : certains, déjà bien qualifiés, doivent recevoir une formation adéquate qu'ils transmettront ensuite à leur entourage. Le Muséum national d'Histoire naturelle peut ici jouer un

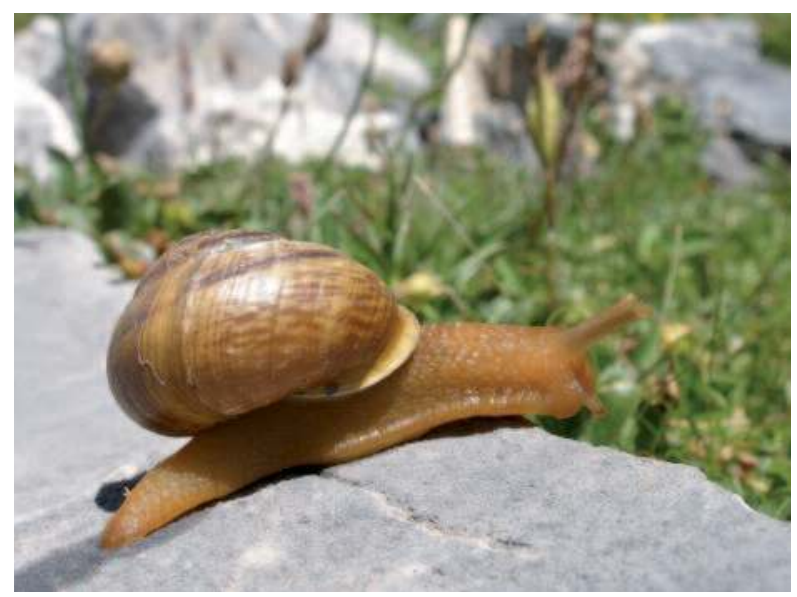

« 50000 observations pour la forêt »: I'Hélice des bois ( ) O. Gargominy/SPN-MNHN rôle déterminant, il a déjà l'habitude dans ses activités d'enseignement et de « recyclage » d'enseignants. Il est clair, ensuite, que le relais doit être pris au niveau associatif (voir ABC, linventaire des communes de France).

Un autre aspect essentiel qui n'a pas encore été abordé dans le cadre de ce rapport, est la situation dans les territoires français outre-mer, sièges d'une extraordinaire diversité en espèces et en habitats et pour lesquels l'effort «participatif » n'est pas encore suffisamment développé. Des associations locales existent parfois mais ont souvent besoin d'aides financières et de contacts avec leurs homologues métropolitains. Ceci est à développer, à encourager et à beaucoup mieux organiser sous l'égide tant de l'État que des collectivités territoriales. En effet, la participation est probablement (cela dépend quand même des territoires) encore plus à organiser qu'en France : les territoires peuvent être très vastes, peu peuplés parfois (Calédonie ou Guyane), ou à l'inverse subir une surdensité de population sur très peu d'espace (Réunion, Antilles...). Les grands organismes de recherche sont moins présents et les universités locales (c'est aussi vrai en métropole) n'en sont qu'aux prémices de contacts avec les sciences participatives. Nous devrons donc mettre en œuvre des mesures particulières pour générer et organiser l'effort participatif dans ces territoires.

Par ailleurs, les taxonomistes «non-professionnels » (scientifiques retraités et " amateurs bénévoles éclairés ») ont été à l'origine de plus de la moitié des espèces d'animaux décrites entre 1998 et 2007 (Silvertown, 2010 ; Fontaine et al., 2010). En Europe, 700 nouvelles espèces sont décrites actuellement par an, 4 fois plus vite quil y a deux siècles. Il s'organise en fait une coopération entre les chercheurs qui utilisent les approches moléculaires du "barcoding » (système international de nomenclature et de phylogénie sur des caractères de séquences de gènes), et les " participatifs » toujours très attachés aux aspects morphologiques. Ils sont toujours indispensables. Durant les «Grandes expéditions » (voir le site de « La planète revisitée » du Muséum national d'Histoire naturelle, de l'Institut de Recherche pour le Développement et de ProNatura International), entre autres, « Santo 2006 », « MozamGascar 2008-2009 », puis actuellement « Madang 2012 », un appel non négligeable est fait aux nonprofessionnels pour la collecte sur le terrain et la détermination ou la description des spécimens ramenés. Une très intéressante initiative a été lancée (Blangy et al., 2011), dénommée ABORINET, pour promouvoir une collaboration étroite entre des peuples indigènes et des chercheurs pour mieux organiser, planifier, gérer 
intelligemment les activités touristiques, vers des zones plus ou moins « sensibles » : en fait une " géocollaboration ». Comment un écotourisme bien pensé peut-il à la fois ne pas trop « déranger » des écosystèmes avec les populations humaines et non-humaines qui y vivent, leur permettre de se développer harmonieusement et durablement, aider les humains autochtones à mieux communiquer et partager entre eux et à permettre la collecte de données utiles à tous pour mieux gérer ? Certaines activités touristiques non contrôlées, par exemple à destination de petites îles (Bœuf, 2011), commencent à poser de sérieux problèmes environnementaux et sociaux.

En fait, le but essentiel de la «participation » au sens large est double:

- avoir accès à des données non exploitables sans une couverture très étoffée des territoires ou des saisons ;

- développer un outil de sensibilisation du public pour amener les observateurs à changer leur regard sur la nature et sur la biodiversité et donc, contribuer collectivement à une bien meilleure prise en charge de la gestion raisonnée et soutenable des écosystèmes. Il est clair qu'un tel objet doit prendre racine et être relayé par l'éducation scolaire, et dès le plus jeune âge, avec un intérêt fortement accru pour les sciences du vivant et de l'environnement.

Finalement, la question finale pourrait-être « ... faudrait-il légiférer ou réglementer (décrets, arrêtés...) les divers modes de participation du public aux sciences (participatives, citoyennes, collaboratives...) ?». Nous ne le pensons pas, car il faut à tout prix conserver le

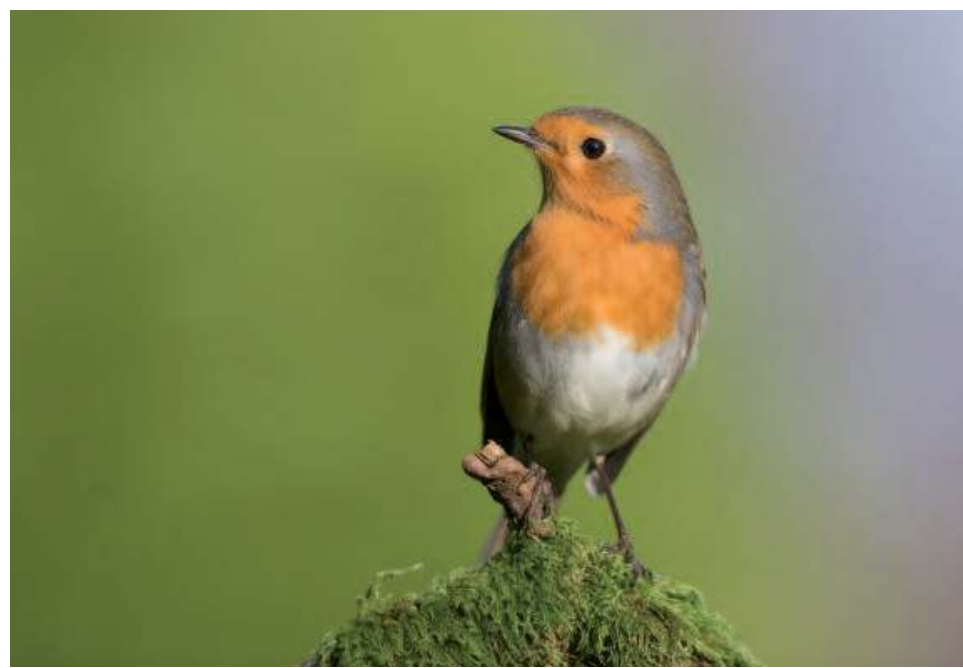

Rester vigilant pour la sauvegarde de la biodiversité. (c) Émile Barbelette/LPO côté « spontané » d'un certain nombre d'initiatives et ne pas « brimer » l'esprit novateur ou innovant par des contraintes réglementaires qui ne pourront que difficilement suivre l'évolution des besoins et des innovations. Néanmoins, ne faudrait-il pas trouver une méthode de validation des programmes lancés par des laboratoires appartenant à des institutions publiques, voire privées, surtout quand des fonds tant publics que privés sont également recherchés ? Il nous apparaît aussi nécessaire que les diverses initiatives, surtout quand elles prétendent faire de la science participative de manière ouverte, puissent être «enregistrées » quelque part. À l'instar du système en place aux ÉtatsUnis avec la Citizen Science Alliance, une coordination générale et centralisée pourrait être envisagée. Ceci rendrait service au ministère de l'Écologie mais est à débattre avec tous les partenaires, le seul but étant de réellement améliorer la situation actuelle, non de la fragiliser ou de la rendre inopérante.

\section{Note}

(1) Cet article est le condensé d'un rapport remis à la ministre de l'Écologie en janvier 2012 : Bøuf, G., Allain, Y.-M. et Bouvier, M. L'apport des sciences participatives à la connaissance de la biodiversité. Rapport du ministère de l'Écologie, du Développement Durable, des Transports et du Logement, 2012, 29 p.

\section{Bibliographie}

Barbault, R. Un éléphant dans un jeu de quilles. L'homme dans la biodiversité. Paris : Seuil, Science ouverte, 2006, 266 p.

Barnosky, A.-D. et al. Has the Earth's sixth mass extinction already arrived ? Nature, ${ }^{\circ} 471,2011$, pp. 51-57.

Barriopedro, D., Fischer, E.-M., Lüterbacher, J., Trigo, R.-M. et GarciaHerrera, R. The hot summer of 2010 : redrawing the temperature record map of Europe. Science, n³32, 201 1, pp. 220-224.

Blangy, S., Donohoe H.-M. et Mitchell, S. Developing a geocollaboratory for indigenous tourism research. Current Issues in Tourism, 2011, 13 p.

Blondel, J. Biodiversité et sciences de la nature. Les biodiversités, objets, théories, pratiques. Paris: CNRS Éditions, 2005, pp. 23-36.

Blondel, J., Aronson, J., Bodiou, J.-Y. and Boeuf, G. The Mediterranean region, biodiversity in space and time. New York: Oxford University Press, $2010,376 \mathrm{p}$ 
Bœuf, G. Quel avenir pour la Biodiversité ? in Changeux, J.-P. et Reisse, J. (dir.) Un monde meilleur pour tous, projet réaliste ou rêve insensé ? Collège de France/Odile Jacob, 2008, pp. 47-98.

Bœuf, G. Quelle Terre allons-nous laisser à nos enfants ? in Gouyon, P.Y. et Leriche, H. Aux origines de l'environnement. Paris : Éditions Fayard, 2010, pp. 432-445.

Bœuf, G. Marine biodiversity characteristics, C R Biologies, n³34, 2011, pp. 435-440.

Bœuf, G. Île de Pâques, un second effondrement ? Espèces, n², 2011, pp. 66-69.

Butchart, S.H.M. et al., 2010. Global biodiversity : indicators of recent declines. Science, n³28, 1164-1168.

Charvolin, F. Comment penser les sciences naturalistes « à amateurs » à partir des passions cognitives ? Natures, Sciences, Sociétés, n¹7, 2009, pp. 145-154.

Couvet, D., Jiguet, F., Julliard, R., Levrel, H. et Teyssedre, A. Enhancing citizen contribution to biodiversity science and public policy, Interdisciplinary Science Reviews, n³3 (1), 2008, pp. 95-103.

Couvet, D., Devictor, V., Jiguet, F. et Julliard, R. Scientific contributions of extensive biodiversity monitoring, C R Biologies, n³34, 201 1, pp. 370377.

CSPNB La biodiversité à travers des exemples. Ministère de l'Écologie et du Développement durable, MEDD/D4E, 2007, 104 p.

CSPNB La biodiversité à travers des exemples, les réseaux de la vie, tome 2 . Ministère de l'Écologie, de l'Énergie, du Développement durable et de l'Aménagement du Territoire, MEEDDAT, 2008, 196 p.

CSPNB La biodiversité à travers des exemples, services compris, tome 3. Ministère de l'Écologie, du Développement durable et de l'Énergie, MEDDE, 2012, 184 p.

Crutzen, P.-J. et Stoermer, E.-F. The Anthropocene, Global Change Newsletter, $\mathrm{n}^{\circ} 41,2000$, pp. 12-13.

Deguines, N., Julliard, R., de Flores, M. et Fontaine, C. The whereabouts of flower visitors : contrasting land-use preferences revealed by a countrywide survey based on citizen science, PLoS ONE, 2012 (sous presse).

Devictor, V., Whittaker, R.-J. et Beltrame, C. Beyond scarcity : citizen science programmes as useful tools for conservation biogeography. Diversity and Distributions, $\mathrm{n}^{\circ} 16$ (3), 2010, pp. 353-362.

Devictor, V. et al. Differences in the climatic debts of birds and butterflies at a continental scale, Nature Climate Change, 2012, 4 p.
Fontaine, B. et al. European bounty for taxonomists, Nature, ${ }^{\circ} 468$, 2010 , p. 377.

Gosselin, M., Gosselin, F. et Julliard, R. L'essor des sciences participatives pour le suivi de la biodiversité, intérêts et limites. Science, eau et territoires, $\mathrm{n}^{\circ} 3,2010,15 \mathrm{p}$

Happe, D. Biodiversité et science paticipative. Présentation à la Journée «Biodiversité » du 10 septembre 2010 aux agents DDT53 en Mayenne.

He, F. et Hubbell, S.-P. Species area relationships always overestimate extinction rates from habitat loss, Nature, $n^{\circ} 473,2011$, pp. 368-371.

IFFRÉE Sciences participatives et biodiversité. Les livrets de l'IFRÉE, $\mathrm{n}^{\circ} 2$, 2011,107 p.

Julliard, R. Entretien sur l'apport des sciences participatives à la biodiversité. Paris : Muséum national d'Histoire naturelle, septembre 2010.

Legrand, M. Programmes de sciences participatives et initiatives apparentées sur le thème de la biodiversité en France. Mémoire du MNHN, 2010.

Le Maho, Y. et Boucher, J. Mission de réflexion sur l'organisation française en matière d'expertise sur la biodiversité. Rapport du MEDDT, 201 1, 23 p.

Lévêque, C. et Mounolou, J.-C. Biodiversité. Dynamique biologique et conservation. Dunod, 2001, 248 p.

Levrel, H., Fontaine, B. Henry, P.-H., Jiguet, F., Julliard, R., Kerbiriou, C. et Couvet, D. Balancing state and volunteer investment in biodiversity monitoring for the implementation of CBD indicators ; a French example. Ecological Economics, n69, 2010, pp. 1580-1586.

Mathieu, D. Réseaux collaboratifs et sciences participatives. Conférence de Tela Botanica, 2010, $18 \mathrm{p}$.

Mathieu, D. Observer la nature, une problématique « science citoyenne »? Colloque «Observer et s'adapter aux changements climatiques en forêt méditerranéenne », Marseille, 30 novembre-3 décembre 2010.

Millennium Ecosystem Assessment Ecosystems and human well-being : synthesis. Washington DC : Island Press, 2005, 137 p.

Mora, C. et al. How many species are there on the Earth and in the ocean? PLoS Biology, n9, 2001

Palumbi, S.-R. Humans as the world's greatest evolutionary force. Science, n²93, 2001, pp. 1786-1790.

Rey Benayas, J.-M., Newton, A.-C., Diaz, A. et Bullock, J.-M. Enhancement of biodiversity and ecosystem services by ecological restoration : a meta-analysis. Science, n²35, 2009, pp. 1121-1124. 
Silvertown, J. Taxonomy : include social networking. Nature, ${ }^{\circ} 467$, 2010 , p. 788

Tela Botanica Observons la nature, des réseaux et des sciences pour préserver la biodiversité. $2009,58 \mathrm{p}$

Thomas, C.-D. et al. Extinction risk from climate change. Nature, $\mathrm{n}^{\circ} 427$, 2004, pp.145-148.

Vilá, M. et al. Ecological impacts of invasive alien plants : a meta-analysis of their effects on species, communities and ecosystems. Ecology Letters, 2011.

\section{Sites Internet}

STOC : http://vigienature.mnhn.fr/page/le-suivi-temporel-des-oiseauxcommuns-stoc

SPIPOLL : www.spipoll.org

Vigie-Nature : http://vigienature.mnhn.fr/ 